\title{
Coagulation Abnormalities Following NexoBrid Use: A Case Report
}

\author{
Nuria Martín MD, * Patricia Guilabert MD, * Luis Abarca MD, * \\ Gemma $M^{a}$ Usua $M D,{ }^{*}$ Jordi Serracanta $M D,{ }^{\dagger}$ Maria J. Colomina, $\mathrm{MD}, \mathrm{PhD}^{\ddagger}$
}

\begin{abstract}
Patients with major burn injury undergo a series of pathophysiologic changes that begin with a systemic inflammatory response and coagulation abnormalities, similar to those experienced by patients with sepsis or severe trauma. Coagulation changes in patients with burns are generally characterized by procoagulant abnormalities, but alterations in fibrinolysis and anticoagulation factors have also been observed. Around $40 \%$ of patients with major burn show changes on standard coagulation tests, and these have been related to the severity of the lesions, smoke inhalation, and administration of intensive fluid resuscitation therapy. Current surgical techniques for debridement of burn lesions are aggressive and associated with considerable blood loss. A fast-acting selective enzymatic debriding agent based on bromelain has been recently developed. NexoBrid is indicated for removing eschar in adults with deep partial- and full-thickness thermal burns. A potential effect of oral bromelain on hemostasis has been described, but it is uncertain whether NexoBrid application has a clinically relevant impact in this regard. We present the clinical case of a patient with burns who showed a coagulation abnormality shortly after NexoBrid use.
\end{abstract}

Patients with major burn injuries experience a series of pathophysiologic changes, beginning with a systemic inflammatory response ${ }^{1}$ and coagulation abnormalities that resemble what occurs in patients with sepsis or severe traumatic injury. The coagulation disturbance in patients with burn is characterized by procoagulant changes, but abnormalities in fibrinolysis and factors related with anticoagulation have also been observed. ${ }^{2}$ Overall, around $40 \%$ of patients with major burn show changes (most often the prothrombin time) on standard coagulation testing in the first 24 hours. These abnormalities are related to the severity of the burn, smoke inhalation, and administration of intensive fluid therapy. ${ }^{3-5}$

\footnotetext{
*Anesthesia and Critical Care Department and Burn Center and

† Plastic Surgery Department and Burn Centre, University Hospital Vall d'Hebron, Barcelona, Spain and ${ }^{\ddagger}$ Anesthesia and Critical Care Department, Bellvitge University Hospital, Universitat de Barcelona, Spain.

Address correspondence to Nuria Martín Marata, MD, Anesthesia and Critical Care Department and Burn Center, Hospital Universitari Vall d'Hebron, Passeig de la Vall d'Hebron 119-129, Barcelona 08035, Spain. Email: nmartinmarata@gmail.com

(C) American Burn Association 2018. All rights reserved. For permissions, please e-mail: journals.permissions@oup.com 1559-047X/2017

doi:10.1093/jbcr/irx044
}

Current surgical techniques for burn wound debridement are associated with considerable blood loss due to the aggressive action on tissues. To improve this situation, a fast-acting, selective enzymatic debridement agent based on bromelain was recently developed (NexoBrid; MediWound Ltd, Israel). This product is indicated for removing eschar in adults with deep partial- and full-thickness thermal burns. As compared to the standard of care, surgical debridement, enzymatic debridement seems to result in a lower procedure-related blood loss. ${ }^{6}$ However, NexoBrid has been implemented in clinical practice only recently, and there is little information regarding its management, safety, and efficacy. According to the product insert, orally administered bromelain may have an effect on hemostasis, but it is unknown whether NexoBrid application could have a clinically relevant impact in this regard, ${ }^{7}$ such as an increased risk of bleeding. During clinical development of the product, there was no evidence indicating this potential effect.

Studies published by Rosenberg et al, in 2014 and 2015 , found no coagulation-related adverse effects in patients receiving NexoBrid, ${ }^{8}$ and the only reference to analytical parameters was in relation to bleeding, where 
it was concluded that the drop in hemoglobin and hematocrit was smaller in patients receiving NexoBrid than in those undergoing surgical debridement. ${ }^{6}$

NexoBrid is applied on the burn and left to act for a period of 4 hours. Effective analgesia is required while it is being applied, during the time it acts, and when it is removed. Locoregional anesthesia or deep sedation can be used for this purpose, depending on the magnitude of the lesions sustained. Here, we report the case of a patient with burn injury who experienced a coagulation abnormality following NexoBrid use.

\section{CLINICAL CASE}

A 41-year-old man, weighing $89 \mathrm{~kg}$, tobacco and cannabis smoker, reporting moderate alcohol consumption, occasional cocaine use, and no medication allergies or medical history of interest, sustained a burn injury caused by an industrial aerosol explosion in the factory where he was working. At the initial evaluation, the emergency services noted facial burns and dysphonia. Orotracheal intubation was performed, which disclosed mild glottic edema. After the airway had been secured, the patient was transferred to our center in a state of hemodynamic and respiratory stability. Before arriving at our hospital, he received $2000 \mathrm{ml}$ of crystalloid infusion.

On admission, the total extent of the burn injury was estimated using the Lund and Browder chart (Figure 1). The patient had burns on $15 \%$ of TBSA, located on the forearms and hands, upper third of the face and scalp, and both knees. The lesions were classified as mainly intermediate/deep second-degree burns with some areas of erythema on the lower third of the face and anterior cervical region. There were no third-degree burn areas.
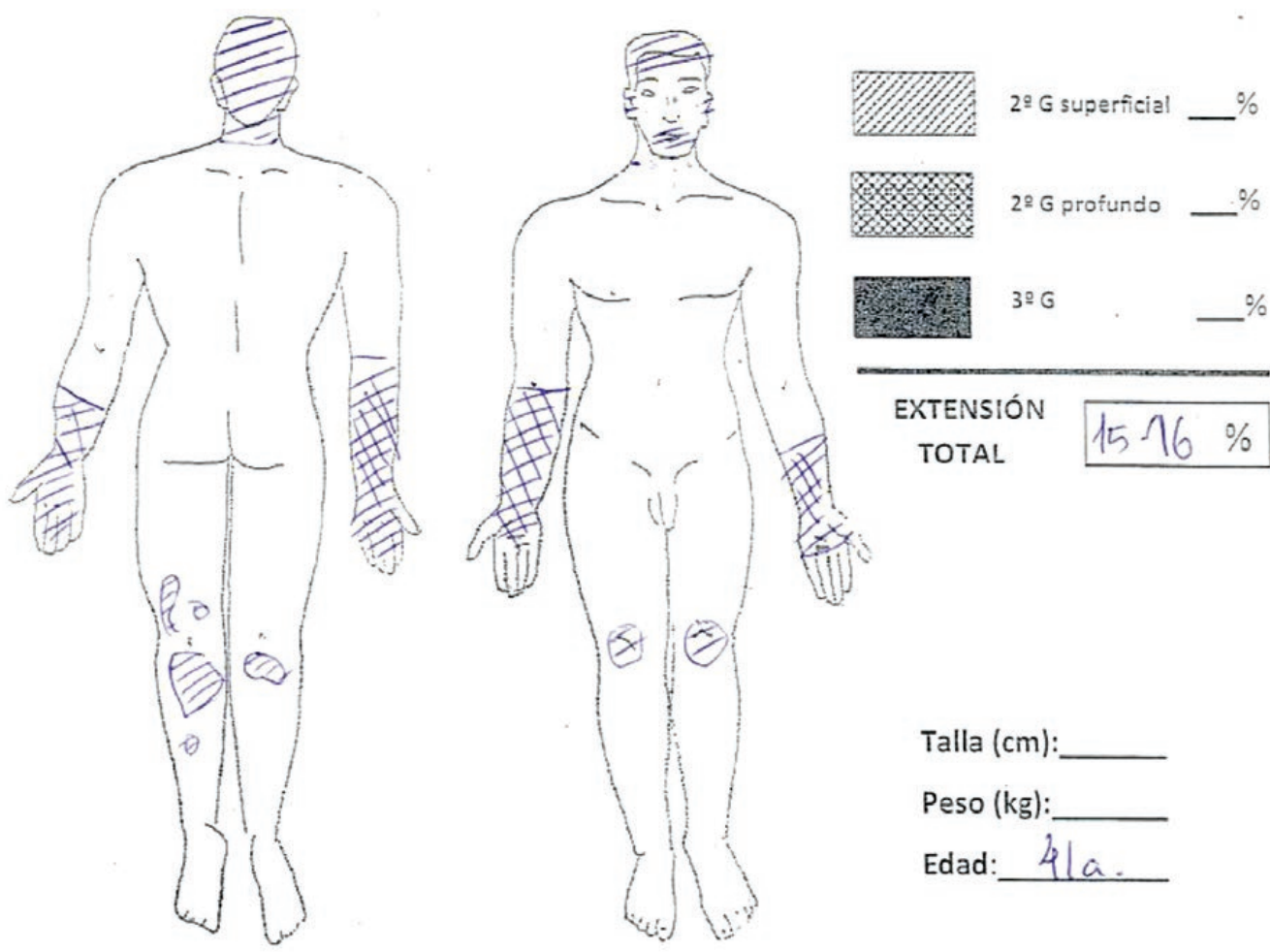

Superficie Corporal Total quemada (SCT)

\begin{tabular}{|c|c|c|}
\hline Cabeza ....................... 7\% & Brazos ……............. $8 \%$ & Nalgas ........................ $5 \%$ \\
\hline Cuello ......................... $2 \%$ & Antebrazos ................ $6 \%$ & Musios .................... 19\% \\
\hline Tórax y abdomen .......13\% & Manos ..................... $5 \%$ & Piernas ...................... 14\% \\
\hline Espalda .............................. & Genitales & Pies …................. 7\% \\
\hline
\end{tabular}

Figure 1. Lund and Browder chart for adults 
Table 1. Analytic determinations

\begin{tabular}{lcccccc}
\hline & $\begin{array}{c}\text { Hemoglobin } \\
(\mathrm{g} / \mathrm{dl})\end{array}$ & $\begin{array}{c}\text { Platelet Count } \\
\left(\times 10^{6} / \mathbf{l}\right)\end{array}$ & PT (\%) & PT Ratio & aPTT Ratio & Fibrinogen $(\mathrm{g} / \mathbf{l})$ \\
\hline 29 October (Day 1, before & 15 & 165,000 & 76 & 1.19 & 1.01 & 3.58 \\
$\quad$ NexoBrid use) & & & & & & \\
30 October, 8:56 am & 9.8 & 110,000 & 43 & 1.78 & 1.24 & 3.98 \\
30 October, 11:50 am & & & 47 & 1.74 & 1.1 & 4.64 \\
31 October & 10.5 & 126,000 & 80 & 1.16 & 0.94 & 5.85 \\
1 November & 9.7 & 149,000 & 87 & 1.10 & 0.81 & 5.86 \\
\hline
\end{tabular}

aPTT, activated partial thromboplastin time; PT, prothrombin time.

The calculated Acute Burn Severity Index was 7, and the estimated risk of death was between 10 and $20 \%$. Analytic determinations on admission showed no abnormalities (Table 1). Initial fluid resuscitation was performed according to the Parkland formula, corrected to maintain diuresis above $0.5 \mathrm{ml} / \mathrm{kg} / \mathrm{h}$. The patient received $1600 \mathrm{ml}$ of balanced crystalloid (Ringer's lactate) in the first 6 hours.

On day 1 following admission and based on the characteristics of the burns, it was decided to perform NexoBrid enzymatic debridement on the forearms, the areas with the deepest burns, accounting for $6 \%$ of the TBSA. As the patient was still intubated, it was only necessary to deepen the sedation and analgesia to perform the enzymatic debridement technique. The product was applied and left in place for 4 hours. Two hours after its removal, the patient showed central hyperthermia of $40^{\circ} \mathrm{C}$ without hemodynamic instability. In response to this development, blood, urine, and tracheal aspirate samples were taken for culture, and antibiotic therapy was started, even though the hyperthermia could have been related to NexoBrid application. ${ }^{7}$

Analytic controls performed at 24 hours after debridement disclosed decreased coagulation activity: prothrombin time ratio 1.78 and activated partial thromboplastin time ratio 1.24. The patient showed no signs of active bleeding, platelet count had dropped somewhat $\left(110,000 \times 10^{6} / 1\right)$, and hemoglobin measured $9.8 \mathrm{~g} / \mathrm{dl}$. Because of the discrepancy relative to the baseline values, the analysis was repeated, and the changes were confirmed. Intravenous single dose of 10-mg phytomenadione was then administered, and in 24 hours, the coagulation parameters had returned to normal values. The patient showed no other coagulation changes in the following analytic determinations. Since the time of hospital admission, $9840 \mathrm{ml}$ of crystalloids and $800 \mathrm{ml}$ of albumin $20 \%$ had been infused.

Initial extubation could not be performed due to hypoxemia secondary to left lung basal atelectasia. After respiratory therapy, the patient could be extubated on day 4 following admission, showing mild, clinically well-tolerated postextubation hypoxemia. Chest radiographs showed signs of vascular redistribution with bilateral infiltrates, and the tracheal aspirate tested positive for cloxacillin-sensitive Staphylococcus aureus. No other lung injuries were found. A diagnosis of tracheobronchitis and suspected smoke inhalation was established, and combined treatment with antibiotics, diuretics, and Optiflow oxygen therapy was administered. The hypoxemia gradually improved and had resolved at 7 days. The total positive fluid balance was $17.239 \mathrm{ml}$. The patient additionally received antibiotics for a central venous line infection and chlormethiazole for alcohol abstinence. His clinical course was favorable, and he was discharged on day 16 following admission.

\section{LITERATURE REVIEW OF BROMELAIN USE}

A PubMed search was performed, and four articles related to NexoBrid use were retrieved. ${ }^{6},{ }^{8-10} \mathrm{We}$ excluded one article ${ }^{9}$ because it focused on the use of a bromelain derivate for enzymatic fasciotomy in Dupuytren's disease. Among the remaining three articles, the most recent one reported the clinical case of a 14-year-old patient satisfactorily treated with NexoBrid after sustaining a burn injury due to explosion of the lithium battery in his mobile phone. No treatment-related adverse effects were detected. ${ }^{10}$

Rosenberg et al published a multicenter phase-3 clinical trial including 26 centers in 13 different countries. ${ }^{8}$ Patients 4 to 44 years of age with burns covering 5 to $30 \%$ of BSA were included. The results showed no coagulation-related adverse effects.

In their study in 2015, the same authors reviewed the findings of seven clinical studies reporting NexoBrid use. ${ }^{6}$ They concluded that enzymatic debridement completely eliminates eschar without injuring surrounding tissues, exposing a viable dermis bed. In addition, it was found to reduce the increase in interstitial compartment pressure in circumferential 
burns of the extremities. No reference to coagulation abnormalities was made in this second study.

\section{DISCUSSION}

Coagulation changes are not uncommon in patients with burn injury. Acute burn-induced coagulopathy is a well-described entity similar to the acute coagulopathy occurring in patients with trauma. According to Sherren et al, ${ }^{3}$ around $40 \%$ of patients with major burn have coagulopathy at hospital admission, which correlates with the severity of the burn and is associated with the extension of the injury, smoke inhalation, the Acute Burn Severity Index, and the total burn thickness.

The patient presented here had burns more than $15 \%$ of TBSA; hence, he cannot be considered a patient with major burn. He would have a low probability of showing coagulation abnormalities related to the thermal lesion, and this was supported by the normal values found in the analyses at admittance. Smoke inhalation is another factor favoring acute burn-induced coagulopathy. In our clinical experience, patients who experience smoke inhalation have increased fluid requirements due to the lesion itself, and this could lead to dilution coagulopathy. Nonetheless, although our patient had an inhalation lesion, he did not require more than $4.4 \mathrm{ml} / \mathrm{kg} / \mathrm{h}$ of fluids in the first 24 hours, an amount close to the value given in standard protocols and which should not lead to dilution coagulopathy.

Another cause of justifiable coagulopathy would have been a septic state but other than atelectasia and tracheobronchitis, no other lung or systemic injuries were found to qualify our patient as septic.

The acute coagulation abnormality exhibited by our patient manifested soon after treatment with NexoBrid, and this could be interpreted as a causal relationship. In the product insert, coagulopathy is mentioned as a potential adverse effect of NexoBrid use, but it has not been described in any of the patients treated with this agent. Reduced platelet aggregation and plasma fibrinogen concentrations, and moderately increased prothrombin and partial thromboplastin times have been reported as possible effects following oral bromelain administration. Data from in vitro and experimental animal studies suggest that bromelain may also enhance fibrinolysis. During the clinical development phase of NexoBrid, there were no findings to indicate an increased risk of bleeding at the debridement site, ${ }^{7}$ but the study made no reference to analytic changes. In our unit, viscoelastic techniques were not available to further evaluate the analytic abnormalities observed.

Our patient additionally showed hyperthermia following enzymatic debridement. We can reasonably discard a systemic cause for this, as tracheobronchitis and central line infection were localized. Operating room conditions might also be the cause of hyperthermia, but our patient never required surgery. In this case, we attribute the hyperthermia to NexoBrid itself, as is also described in the product insert. The mechanism causing this effect is unknown.

In light of the observations in our patient, we believe it may be advisable to monitor possible coagulation-related adverse effects in patients treated with NexoBrid. It may also be appropriate to use this product with caution in patients receiving antiplatelet or anticoagulant therapy, particularly if locoregional anesthesia techniques are used for performing the enzyme debridement technique.

Conflict of interest statement. None declared.

\section{REFERENCES}

1. Guilabert P, Usúa G, Martín N, Abarca L, Barret JP, Colomina MJ. Fluid resuscitation management in patients with burns: update. Br J Anaesth 2016;117:284-96.

2. Glas GJ, Levi M, Schultz MJ. Coagulopathy and its management in patients with severe burns. J Thromb Haemost 2016;14:865-74

3. Sherren PB, Hussey J, Martin R, Kundishora T, Parker M, Emerson B. Acute burn induced coagulopathy. Burns 2013;39:1157-61.

4. King DR, Namias N, Andrews DM. Coagulation abnor malities following thermal injury. Blood Coagul Fibrinolysis 2010;21:666-9.

5. Lavrentieva A, Kontakiotis T, Bitzani M et al. Early coagulation disorders after severe burn injury: impact on mortality. Intensive Care Med 2008;34:700-6.

6. Rosenberg L, Shoham Y, Krieger Y et al. Minimally invasive burn care: a review of seven clinical studies of rapid and selective debridement using a bromelain-based debriding enzyme $\left(\right.$ Nexobrid $\left.^{\circledR}\right)$. Ann Burns Fire Disasters 2015;28:264-74

7. European Medicines Agency. Nexobrid Summary of product characteristics. London, UK: European Medicines Agency. 2006. p. 809-22 accessed 2017; available from http://www.ema.europa.eu/docs/en_GB/document_library/EPAR_-_Product_Information/human/002246/ WC500136580.pdf.

8. Rosenberg L, Krieger Y, Bogdanov-Berezovski A, Silberstein E, Shoham Y, Singer AJ. A novel rapid and selective enzymatic debridement agent for burn wound management: a multi-center RCT. Burns 2014;40:466-74.

9. Rubin G, Rinott M, Wolovelsky A, Rosenberg L, Shoham Y, Rozen N. A new bromelain-based enzyme for the release of Dupuytren's contracture: Dupuytren's enzymatic bromelain-based release. Bone Joint Res 2016;5:175-7.

10. Cherubino M, Pellegatta I, Sallam D, Pulerà E, Valdatta L. Enzymatic debridement after mobile phone explosion: a case report. Ann Burns Fire Disasters 2016;29:273-5. 